\title{
Design of a Direct Conversion Transmitter to Resist Combined Effects of Power Amplifier Distortion and Local Oscillator Pulling
}

\author{
Chieh-Hsun Hsiao, Student Member, IEEE, Chi-Tsan Chen, Student Member, IEEE, \\ Tzyy-Sheng Horng, Senior Member, IEEE, and Kang-Chun Peng, Member, IEEE
}

\begin{abstract}
This work elucidates how the combined effects of power-amplifier distortion and local-oscillator (LO) pulling adversely impact a wireless direct-conversion transmitter (DCT) that adopts a time-varying envelope modulation. An analytical model is developed to evaluate the deterioration of DCT output signal quality, including error vector magnitude, adjacent channel power ratio, and spectral regrowth. Additionally, an integrated approach for PA linearization and anti-LO pulling is designed based on an open-loop digital-predistortion method and the proposed analog feedback compensation mechanism. Experimental results demonstrate that a quadrature-modulation-based DCT that incorporates the proposed approaches can significantly improve the LO spectral purity, while achieving a high linearity and efficiency performance simultaneously. These attributes are highly desired for third-generation systems such as cdma2000 1x and W-CDMA.
\end{abstract}

Index Terms-Digital predistortion (DPD), direct conversion transmitter (DCT), local-oscillator (LO) pulling, power amplifier (PA), PA linearization, transmitted signal quality.

\section{INTRODUCTION}

W IRELESS communications have driven the demand for increased capacity and flexibility of RF transceiver design in recent decades [1], [2]. Achieving high-data-rate signal transmission for multimedia communications, especially in wide modulation bandwidth and high peak-to-average power ratio (PAPR), has led to stringent linearity constraints on signal conversion and amplification [3], [4]. Fig. 1 illustrates a widely adopted quadrature-modulation-based direct-conversion transmitter (DCT) [2], which is characterized by its low modulation distortion, regardless of the data rate. The DCT consists mainly of a local oscillator (LO) to provide a pure sinusoidal signal and a quadrature modulator to perform the baseband IQ modulation, as well as a power amplifier (PA) to boost the transmitted

Manuscript received October 01, 2011; revised March 09, 2012; accepted March 13, 2012. Date of publication April 30, 2012; date of current version May 25,2012 . This work was supported in part by the National Science Council, Taiwan, under Grant 100-2221-E-110-081-MY3, Grant 100-2221-E-110-082 MY3, and Grant 100-2622-E-110-001-CC1.

C.-H. Hsiao, C.-T. Chen, and T.-S. Horng are with the Department of Electrical Engineering, National Sun Yat-Sen University, Kaohsiung 804, Taiwan (e-mail: d983010017@ee.nsysu.edu.tw; D963010023@ee.nsysu.edu.tw; jason@ee.nsysu.edu.tw).

K.-C. Peng is with the Department of Computer and Communication Engineering, National Kaohsiung First University of Science and Technology, Kaohsiung 811, Taiwan (e-mail: peterpkg@ccms.nkfust.edu.tw).

Color versions of one or more of the figures in this paper are available online at http://ieeexplore.ieee.org.

Digital Object Identifier 10.1109/TMTT.2012.2193139

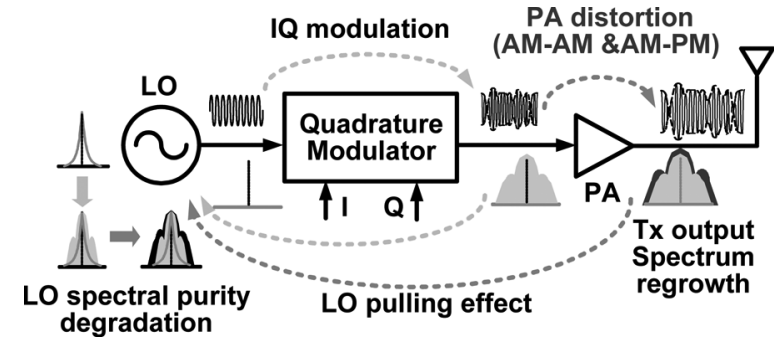

Fig. 1. Illustration of transmitted signal quality degradation owing to combined effects of PA distortion and LO pulling in a DCT.

signal power level. However, when the DCT is operated in a time-varying envelope modulation system, the combined effects of PA distortion and $\mathrm{LO}$ pulling significantly deteriorate the system's signal quality, including the LO spectral impurity and DCT output spectral regrowth. The above problems thus pose major bottlenecks in implementing RF transceiver system-on-chip (SoC).

According to a survey of PA linearization strategies [3]-[7], the digital predistortion (DPD) method is a highly promising solution to improve the PA performance because of its relatively easy programming, low power consumption, and superior linearization. Based on the characteristics of the PA's amplitude-to-amplitude modulation (AM-AM) and amplitude-tophase modulation (AM-PM) distortions, the predistorter implemented in baseband can synthesize the predistorted baseband in-phase/quadrature (IQ) modulation signals to compensate for PA's amplitude compression and phase error. However, as the system modulation bandwidth increases, the DPD methods are generally difficult to improve the transmitted signal quality effectively, due to the limited sampling rate and the excessive quantization noise [5], [7].

The other critical distortion issue, the LO pulling effect, has received considerable attention under various injection conditions. In 2004, Razavi [8] considered a phase-locked oscillator (PLO) under an independent sinusoidal injection. In 2008, Li et al. [9] devised a numerical approach for accurately predicting the LO output spectra and phase noise under an independent modulation injection. While imitating a realistic phenomenon in which an injection signal into the LO correlates with the LO output signal, our previous works [10], [11] introduced a rigorous phase dynamic model for a PLO under directly modulated self-injection to evaluate the degradation of LO phase noise and transmitted signal quality. Moreover, closed-loop analytical models in both frequency domain [12] and time domain 
[13] were developed to analyze the frequency modulation (FM) distortions caused by the LO pulling effect in a time-varying envelope modulation system.

In addition to the above efforts, several works have attempted to mitigate the LO pulling effect. The most common means is to isolate the LO far away from the noisy source with the assistance of the clock retiming approach [14] and bulky off-chip elements [15], which significantly increases hardware complexity and degrades the system integration. A preliminary publication of this work [13] proposed a solution approach by combining a second-point VCO modulation loop and an inner self-injection loop, capable of reducing the LO pulling effect on the transmitted signal distortions. However, if the serious AM-AM and AM-PM distortions of a PA dominate the deterioration of the transmitted signal quality at a high DCT output power, the improved approach in [13] is no longer applicable.

While elucidating the combined effects of PA distortion and LO pulling, this work significantly expands upon the results of [13] by including a detailed analysis of the PA's AM-AM and AM-PM distortions. The rigorous analytical model adopted here can characterize the DCT signal quality deterioration when an $\mathrm{LO}$ is pulled by a self-dependent time-varying envelope modulation signal. This work also develops an improved approach, in which an open-loop DPD, a second-point VCO modulation loop, and an inner self-injection loop are incorporated to enhance the DCT's LO spectral purity, PA linearity, and average efficiency simultaneously.

\section{ANALYSIS APPROACHES}

This section introduces the analytical model for a DCT with combined effects of PA distortion and LO pulling. A time-varying envelope modulation system is also considered when the deterioration of transmitted signal quality is characterized using the proposed model.

\section{A. PA Distortion}

PA distortion originates mainly from two categories of nonlinearities, which are associated with the input signal amplitude [3]. Defined as the nonlinear relation between the amplitudes of the input and output signal, AM-AM distortion often compresses the gain and saturates the output signal amplitude. Conversely, AM-PM distortion describes the output phenomenon in which the PA output phase varies with the input signal amplitude. The above two distortions both induce intermodulation to degrade PA linearity and transmitted signal quality. Consider the time-domain waveforms of the PA's input signal $V_{\text {in }}(t)$ and output signal $V_{\text {out }}(t)$ in a complex form, as expressed in the following equations:

$$
\begin{aligned}
V_{\text {in }}(t) & =E(t) e^{j\left(\omega_{c} t+\phi_{m}(t)\right)} \\
V_{\text {out }}(t) & =G_{i}(E(t)) E(t) e^{j\left(\omega_{c} t+\phi_{m}(t)+\theta_{i}(E(t))\right)}
\end{aligned}
$$

where

$$
\begin{aligned}
E(t) & \propto \sqrt{V_{I}(t)^{2}+V_{Q}(t)^{2}} \\
\phi_{m}(t) & =\tan ^{-1}\left[\frac{V_{Q}(t)}{V_{I}(t)}\right]
\end{aligned}
$$

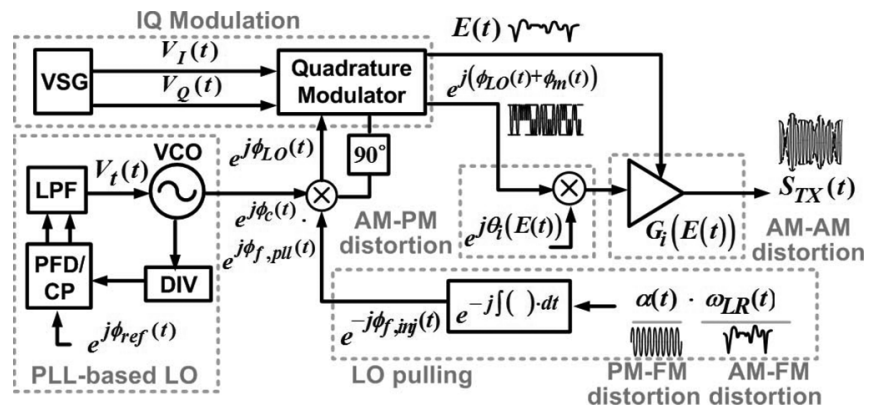

Fig. 2. System model of a DCT with combined effects of LO pulling and PA distortion.

are the envelope and phase modulation (PM) component of the PA's input signal, respectively, based on quadrature modulation of the baseband in-phase $V_{I}(t)$ and quadrature $V_{Q}(t)$ signals. Moreover, $G_{i}(E(t))$ and $\theta_{i}(E(t))$ express the PA's $\mathrm{AM}-\mathrm{AM}$ distortion function and AM-PM distortion function, respectively. Notably, $\omega_{c}$ is the RF carrier frequency.

Fig. 2 illustrates the proposed system model of a DCT with combined effects of PA distortion and LO pulling. The baseband IQ modulation signals are synthesized by a vector signal generator (VSG), and modulated on the RF carrier by a quadrature modulator. A PA is then used to boost the modulated signal power. To accommodate high-data-rate signal transmission, the power back-off is needed to ensure linear operation of the PA [3]. However, for a high PAPR modulation system, a high output back-off (OBO) value must be used accordingly to prevent signal distortion. Unfortunately, the PA's efficiency peaks only when the PA operates near saturation and declines rapidly with a decreasing output power. Under the consideration of excellent linearization and low digital processing complexity, this work implements an open-loop DPD approach in baseband to reduce the PA nonlinear distortions and increase the DCT efficiency [5]-[7], [16].

\section{B. LO Pulling Effect}

As mentioned in Section I, the LO pulling effect has become a major distortion issue with the advances in integrated circuit (IC) process technologies. In the DCT architecture (Fig. 2), the LO is normally implemented by a phase-locked loop (PLL) to control the synthesized signal's frequency and phase precisely, which is achieved by adjusting the voltage-controlled oscillator (VCO) output phase to align with the phase of reference signal $\phi_{\text {ref }}(t)$ through tuning voltage $V_{t}(t)$ and frequency sensitivity $K_{v}$. Based on Adler's analysis [17] and our previous work [13], the instantaneous output frequency of an LO pulled by a timevarying envelope modulation signal can be expressed as

where

$$
\omega_{\mathrm{LO}}(t)=\omega_{\mathrm{osc}}(t) \cdot\left[1-\frac{1}{2 Q} \frac{E_{\mathrm{inj}}(t)}{E_{\mathrm{osc}}} \sin \alpha(t)\right]
$$

$$
\omega_{\mathrm{osc}}(t)=\omega_{c}+K_{v} V_{t}(t)
$$

refers to the inherent oscillation frequency of the VCO and is assumed here to be a summation of synthesized frequency $\omega_{c}$ and the instantaneous frequency variation caused by the PLL. $E_{\text {inj }}(t)$ and $E_{\text {osc }}$ also represent the time-varying and constant 
amplitudes of the injection and oscillation signals, respectively. Meanwhile, $Q$ is the quality factor of the oscillator's tank circuit. Moreover, $\alpha(t)$ is regarded as the instantaneous phase difference between the injection signal and oscillation signal. By assuming that VCO is finally phase locked under a weak narrowband modulation injection, we can infer that $K_{v} V_{t}(t) \ll \omega_{c}$ and $\sin \alpha(t) \approx \alpha(t) \ll 2 Q E_{\mathrm{OSC}} / E_{\mathrm{inj}}(t)$. Therefore, the resulting LO output frequency can be approximated as

$$
\omega_{\mathrm{LO}}(t) \approx \omega_{c}+K_{v} V_{t}(t)-\omega_{\mathrm{LR}}(t) \alpha(t)
$$

where

$$
\omega_{\mathrm{LR}}(t)=\frac{\omega_{c}}{2 Q} \frac{E_{\mathrm{inj}}(t)}{E_{\mathrm{osc}}}
$$

is the time-varying locking range of a free-running oscillator injected by a time-varying envelope modulation signal. In (6), the frequency terms, $K_{v} V_{t}(t)$ and $\omega_{\mathrm{LR}}(t) \alpha(t)$, express the frequency fluctuation caused by the phase- and injection-locking process, respectively. Furthermore, $\omega_{\mathrm{LR}}(t)$ and $\alpha(t)$ represent the amplitude-to-frequency modulation (AM-FM) and phase-to-frequency modulation (PM-FM) distortions that result from the envelope and phase variations, respectively, of an IQ-modulated signal that pulls the LO. Both distortions degrade the quality of IQ modulation in terms of the FM component. Consider a situation in which the injection signal originates from the DCT output modulated signal via the pulling signal injection path with time delay $\tau_{0}$. Based on assumptions of low LO phase noise and narrow modulation bandwidth, the injection-induced PM-FM distortion $\alpha(t)$ can be approximated as

$$
\begin{aligned}
\alpha(t) \approx\left(\phi_{\mathrm{LO}}\left(t-\tau_{o}\right)-\right. & \left.\phi_{\mathrm{LO}}(t)\right) \\
& +\phi_{m}\left(t-\tau_{o}\right)+\theta_{i}\left(E\left(t-\tau_{0}\right)\right)
\end{aligned}
$$

where $\phi_{\mathrm{LO}}(t), \phi_{m}(t)$, and $\theta_{i}(E(t))$ denote the instantaneous phase response of the LO output, IQ modulation, and PA's AM-PM distortion, respectively. Incorporating (8) into (6) and integrating (6) yields the resulting LO output phase

$$
\begin{aligned}
\phi_{\mathrm{LO}}(t) & \approx\left(\phi_{0}+\omega_{c} t\right)+K_{v} \int V_{t}(t) d t-\int \omega_{\mathrm{LR}}(t) \alpha(t) d t \\
& =\phi_{c}(t)+\phi_{f, \mathrm{pll}}(t)-\phi_{f, \mathrm{inj}}(t)
\end{aligned}
$$

where $\phi_{0}$ and $\phi_{c}(t)$ refer to the initial oscillation phase and the synthesized carrier phase, respectively. The phase fluctuation terms, $\phi_{f, \text { pll }}(t)$ and $\phi_{f, \text { inj }}(t)$, in (9) are induced by the phase- and injection-locking process, respectively. A similar phase formulation for the coexistence of phase locking and injection locking can also be found in [14] and [15]. However, this work differs from those works by considering the PA's AM-PM distortion additionally. Based on the above derivations, Fig. 2 illustrates the system model in a complex expression of all signals of interest in the DCT with the combined effects of PA distortion and LO pulling. Consider the LO output signal in the following form:

$$
S_{\mathrm{LO}}(t)=\operatorname{Re}\left\{E_{\mathrm{osc}} e^{j \phi_{\mathrm{LO}}(t)}\right\}=\operatorname{Re}\left\{E_{\mathrm{osc}} e^{j\left(\omega_{c} t+\phi_{f}(t)\right)}\right\}
$$

where $\phi_{f}(t)=\phi_{0}+\phi_{f, \mathrm{pll}}(t)-\phi_{f, \text { inj }}(t)$ is assumed to be the comprehensive phase fluctuation, which originates from the frequency variations of phase- and injection-locking processes. With the discrete-time calculation approach in [9], $\phi_{f}(t)$ can be calculated recursively and formulated as a pulse train weighted by $\phi_{f}[n]$. Therefore, the time-domain waveform of DCT output signal can be expressed as (11), shown at the bottom of this page, where $h_{r}(t)$ denotes the impulse response of a reconstruction filter. This work also characterizes the transmitted signal quality degradation owing to the combined PA distortion and LO pulling effects by assuming that the baseband IQ signal is independent of the LO signal, i.e., $V_{I}(t)$ and $V_{Q}(t)$ are modulated with an uncorrelated LO signal in quadrature modulation.

Finally, incorporating the above equations into Agilent EEsof ADS Ptolemy software to perform system co-simulation allows us to evaluate the degraded transmitted signal quality by some major indices, such as error vector magnitude (EVM), adjacent channel power ratio (ACPR), and spectral regrowth. Fig. 3(a) compares different distortion mechanisms in term of transmitted signal quality degradation, while DCT delivers a 1.98-GHz cdma2000 1x quadrature phase-shift keying (QPSK) modulated signal with a channel bandwidth of $1.25 \mathrm{MHz}$. In this figure, the solid line in blue (in online version), dotted line in red (in online version), and broken line in gray represent the DCT output spectra with no distortion, only PA distortion, and the effects of PA distortion and LO pulling in combination, respectively. The corresponding ACPRs in the same order as above are $-47.2,-37.4$, and $-32.5 \mathrm{~dB}$. Fig. 3(b) shows the demodulated constellation with respect to Fig. 3(a). The corresponding EVMs in the same order as above are $1.3 \%$, $9.4 \%$, and $31.9 \%$.

Above results are simulated when the DCT is operated at an OBO of $6 \mathrm{~dB}$ and a pulling signal power ratio of $-65 \mathrm{~dB}$. The setting of the $\mathrm{OBO}$ is referred to as the PAPR of the synthesized QPSK-modulated signal, while the pulling signal power ratio is defined as the power ratio between the injection signal and the LO output signal (i.e., $E_{\mathrm{inj}, \mathrm{rms}}^{2} / E_{\mathrm{osc}, \mathrm{rms}}^{2}$ ). Above comparisons

$$
\begin{aligned}
S_{\mathrm{TX}}(t) & =\operatorname{Re}\left\{E(t) e^{j\left(\phi_{\mathrm{LO}}(t)+\phi_{m}(t)\right)} \otimes\left[G_{i}(E(t)) e^{j\left(\theta_{i}(E(t))\right)}\right]\right\} \\
& \approx E(t) G_{i}(E(t)) \cos \left[\omega_{c} t+\phi_{m}(t)+\theta_{i}(E(t))+\sum_{n} \phi_{f}[n] h_{r}(t-n T)\right]
\end{aligned}
$$




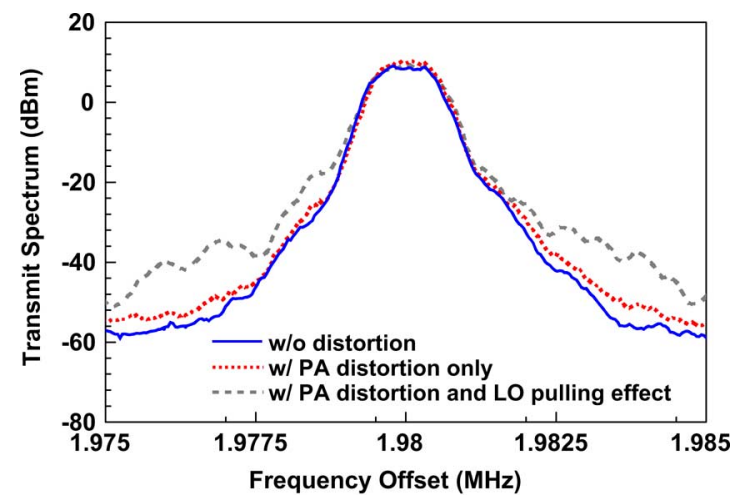

(a)

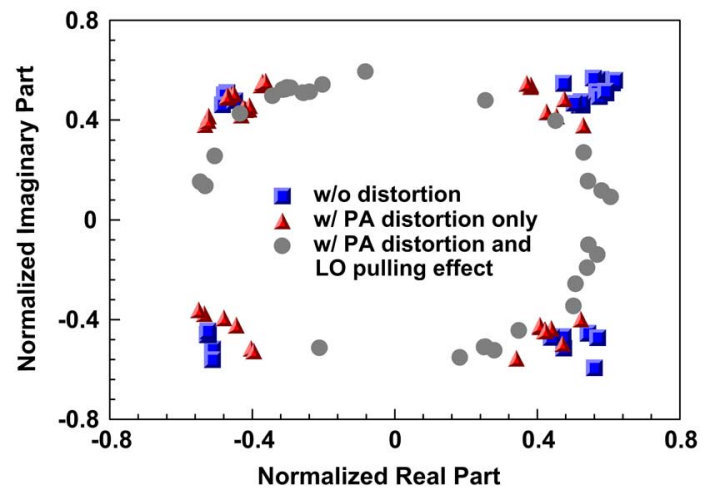

(b)

Fig. 3. Comparison of output signal quality under different distortion mechanisms when DCT delivers a $1.98-\mathrm{GHz}$ QPSK-modulated signal with a 1.25-MHz channel bandwidth. (a) Transmit spectrum. (b) Constellation.

demonstrate that more serious transmitted signal quality degradation originates from the combined effects of PA distortion and LO pulling.

\section{IMPROVEMENT APPROACHES}

Various PA linearization and anti-LO pulling approaches have been developed to alleviate the transmitted signal distortions. Some works [12], [18]-[21] have asserted that the adaptive DPD method may be an effective means of resolving these two major DCT design bottlenecks. With the powerful computation ability of digital circuitry in baseband, a predistorter can provide accurate predistorted values to compensate for the PA's AM-AM and AM-PM distortions through an adaptive calculation algorithm. By exploiting this characteristic, Bashir et al. [12] developed a novel approach for mitigating the LO pulling effect. Therefore, that work introduced an adaptive digital-controlled-delay circuit to break the synchronization between the aggressors and the victims, capable of mitigating the LO pulling effect in the digital polar transmitter architecture.

However, if the PA's nonlinear distortion is considered with the LO pulling effect simultaneously, the serious AM-AM and AM-PM distortions substantially induce a heavy memory load and long convergence time in the adaptive predistortion algorithm. In other words, either the system modulation bandwidth must be reduced or the transmitter $\mathrm{OBO}$ value increased to ensure sufficient improvement on the transmitted signal quality

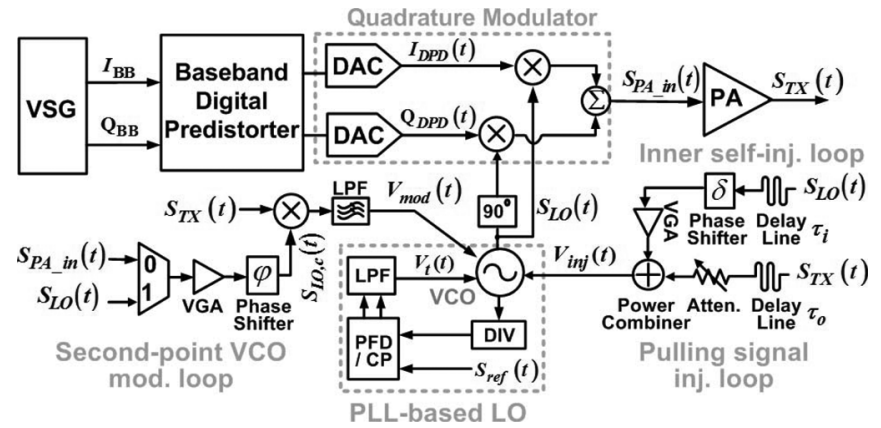

Fig. 4. Block diagram of the proposed transmitter experimental setup for improving the combined effects of LO pulling and PA distortion.

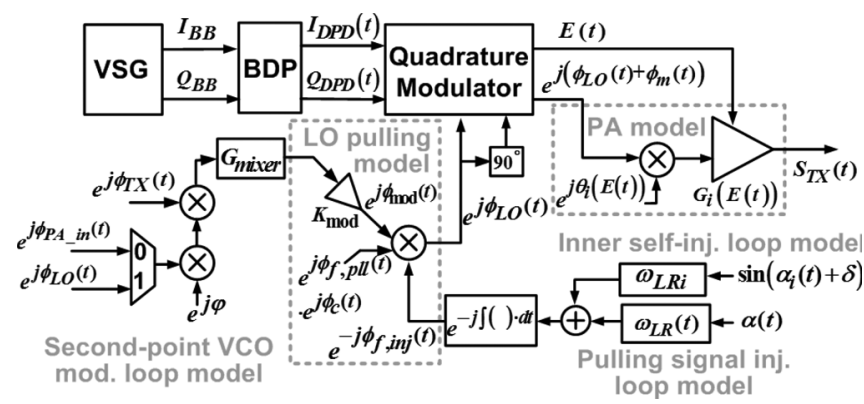

Fig. 5. System model of the experimental DCT shown in Fig. 4.

[21]. However, the transmitter efficiency declines rapidly as the output power decreases.

To overcome the above limitations, this work adopts the open-loop DPD method to reduce the PA's AM-AM and AM-PM distortions. Meanwhile, the proposed second-point VCO modulation and inner self-injection approaches mitigate the LO pulling effect with dependence on the PA's AM-PM distortion.

Fig. 4 schematically depicts the proposed DCT experimental setup, while Fig. 5 illustrates the proposed system model with respect to Fig. 4. The proposed DCT consists mainly of a PLLbased LO, an IQ modulator, a PA, and a baseband digital predistorter (BDP). Baseband I and Q component signals are provided by an Agilent E4438C vector signal generator (VSG) and then predistorted by the BDP. To imitate the real LO pulling phenomenon, a portion of DCT output $S_{\mathrm{TX}}(t)$ is fed back to interfere with LO through a delay line with time delay $\tau_{0}$, an attenuator, and a power combiner, serving as a pulling injection signal. Moreover, with the help of a circulator, the pulling injection signals and the LO output signals can be separated for individual measurement during the experiment, as done in our previous work [11]. In addition to the baseband IQ predistortion process for PA linearization, this work combines the proposed second-point VCO modulation and inner self-injection approaches to reduce the LO spectral impurity incurred by the LO pulling effect.

\section{A. Baseband DPD}

This work attempts to enhance the PA linearity by implementing an open-loop DPD method in baseband [16], [22], [23]. The predistortion concept is described in the following explanation. By continuing with the derivation in Section II-A, 
according to the continuous-wave (CW) measurements of the PA in the absence of DPD, one can characterize the PA's nonlinear functions of gain compression $G_{i}\left(V_{0}\right)$ and phase distortion $P_{i}\left(V_{0}\right)$ with respect to the input sinusoidal phasor $V_{0} e^{j \theta_{0}}$ as follows:

$$
\begin{aligned}
G_{i}\left(V_{0}\right) & =\frac{V_{\text {out }}\left(V_{0}\right)}{V_{0}} \\
P_{i}\left(V_{0}\right) & =e^{j \theta_{i}\left(V_{0}\right)}=e^{j\left(\theta_{\text {out }}\left(V_{0}\right)-\theta_{0}\right)} .
\end{aligned}
$$

Assume that the linearization specifications for the PA's AM-AM and AM-PM distortion are a constant voltage gain of $G_{C}$ and phase difference of zero degree. Therefore, the relative inverse functions, $G_{i}^{-1}\left(V_{0}\right)$ and $P_{i}^{-1}\left(V_{0}\right)$, can be mathematically expressed as

$$
G_{i}^{-1}\left(V_{0}\right)=\frac{G_{c} V_{0}}{V_{\text {out }}\left(V_{0}\right)} \quad P_{i}^{-1}\left(V_{0}\right)=e^{-j\left(\theta_{\text {out }}\left(V_{0}\right)-\theta_{0}\right)} .
$$

Fig. 4 illustrates the approximate implementation of a DPD approach. The baseband in-phase and quadrature component waveforms $I_{B B}$ and $Q_{B B}$ are synthesized by the VSG and fed to the BDP unit for predistortion based on the customized AM-AM and AM-PM lookup tables (LUTs), and subsequently converted into analog waveforms $I_{\mathrm{DPD}}(t)$ and $Q_{\mathrm{DPD}}(t)$ through the digital-to-analog converters (DACs). The predistorted baseband signals are quadrature modulated onto the RF carrier, which forms the input RF signal of the PA. Theoretically, the PA's nonlinear distortions can be effectively reduced with the above predistortion process. However, if the phase shift incurred by the injection-locking effect is considered, the phase error term in (12) should be rewritten as

$$
\theta_{i}\left(V_{0}\right)=\left(\theta_{\text {out }}\left(V_{0}\right)-\theta_{0}\right)-\phi_{f, \text { inj }}\left(V_{0}\right)
$$

where $\phi_{f, \text { inj }}\left(V_{0}\right)$ is an injection-locking phase shift that is dependent on the injection signal amplitude. Obviously, (14) reveals that the transmitted-signal PM distortion originates from the PA's inherent AM-PM distortion and the LO pulling induced FM distortion. Above results also validate our postulation that the open-loop DPD method fails to alleviate the PM distortion of a transmitted signal, owing to the lack of an improvement mechanism for the LO pulling effect.

\section{B. Second-Point VCO Modulation}

An adaptive DPD technique based on feedback concepts has been developed recently to compensate for the inadequate PA linearization of the conventional predistortion approaches. Chung et al. [21] devised an open-loop DPD method embedded with an analog feedback estimation mechanism to expand the system modulation bandwidth and increase the convergence of the predistortion algorithm. The proposed approaches of this work also exploit this characteristic to reduce the PA nonlinear distortion and LO pulling effect simultaneously.

Two equivalent analog feedback mechanisms are proposed in this work, while the distorted envelope and phase signals in the analog form are extracted and fed back to serve as self-adjusting signals to correct the phase error given in (14). Based on the improved results, the proposed approaches significantly contribute efforts to enhance the DCT's LO spectral purity, PA linearity, and average efficiency simultaneously.

As discussed in Section II-B, the serious AM-FM and PM-FM distortions caused by the LO pulling effect significantly degrade the transmitted signal quality in a time-varying envelope modulation system. Moreover, implementing the DPD method should, theoretically, mitigate the PA's AM-AM and AM-PM distortions. However, owing to the limitation of LUT's resolution and PA's physical defects (e.g., thermal noise or memory effect [24]), the transmitted-signal PM distortion is difficult to be cancelled out entirely by using the open-loop DPD method. To eliminate the transmitted signal distortions caused by the PA nonlinear effect in combination with the LO pulling effect, in contrast to the approach of [13], this work offers designing flexibility by introducing a multiplexer in the second-point VCO modulation loop, as shown in Fig. 4. The mixing objects of the RF mixer can be dynamically switched depending on which of the above two effects will dominate.

Firstly, by assuming that the DPD method substantially mitigates the PA's AM-AM distortion, the transmitted-signal PM distortion, caused by the LO pulling induced FM distortion and PA's AM-PM distortion, dominates the transmitted signal quality degradation. Thus, according to Fig. 4, the feedback baseband envelope signal is extracted from the low-frequency mixing product of the DCT output signal $S_{\mathrm{TX}}(t)$ and a calibrated LO signal $S_{\mathrm{LO}, c}(t)$ with the assistance of an RF mixer and a low-pass filter. Mathematically, the extracted baseband envelope signal, $V_{\text {mod }}(t)$, can be expressed as the following equation:

$$
V_{\text {mod }}(t)=\operatorname{LPF}\left\{S_{\mathrm{LO}, c}(t) \otimes S_{\mathrm{TX}}(t)\right\}
$$

where

$$
S_{\mathrm{LO}, c}(t)=G_{\mathrm{VGA}} E_{\mathrm{osc}} \cos \left(\phi_{\mathrm{LO}}(t)+\varphi\right)
$$

and

$$
S_{\mathrm{TX}}(t) \approx G_{c} E(t) \cos \left(\phi_{\mathrm{LO}}(t)+\theta_{i}(E(t))+\phi_{m}(t)\right) .
$$

In the above equations, $\varphi$ and $G_{\mathrm{VGA}}$ denote phase shift and voltage gain of the phase shifter and the variable-gain amplifier (VGA), respectively, which are used in the second-point VCO modulation loop to maximize the conversion gain of the RF mixer. After low-pass filtering out the unwanted frequency items, $V_{\text {mod }}(t)$ can be approximated as (18) under small-angle approximation by incorporating (16) and (17) into (15) when $\varphi=90^{\circ}$, i.e.,

$$
V_{\text {mod }}(t) \approx G_{\text {mixer }} E_{\text {inj }}(t)\left(\theta_{i}(E(t))+\phi_{m}(t)\right)
$$

where $G_{\text {mixer }}$ denotes an equivalent voltage gain associated with the injection signal amplitude $E_{\mathrm{inj}}(t)$. To remove the LO spectral impurity, the extracted baseband envelope $V_{\text {mod }}(t)$ is fed back to the VCO's second modulation point with tuning sensitivity $K_{\text {mod }}$ in order to correct the LO output frequency or phase. Consequently, by incorporating (8) into (6) with consid- 


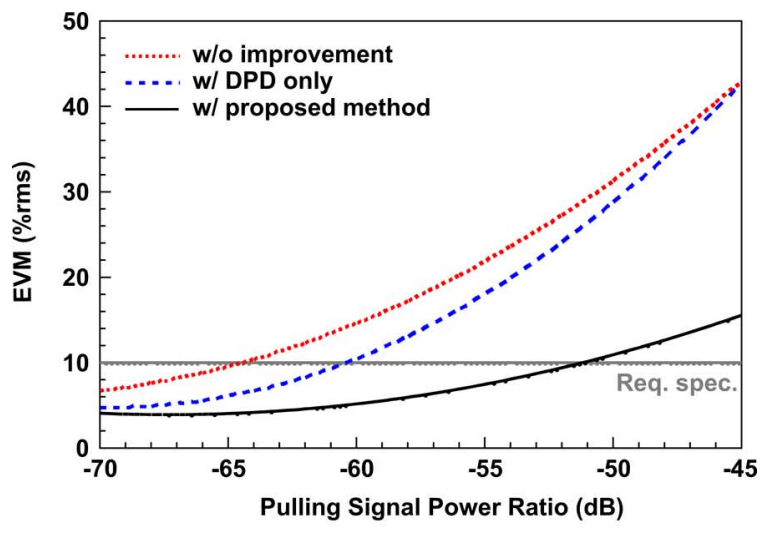

(a)

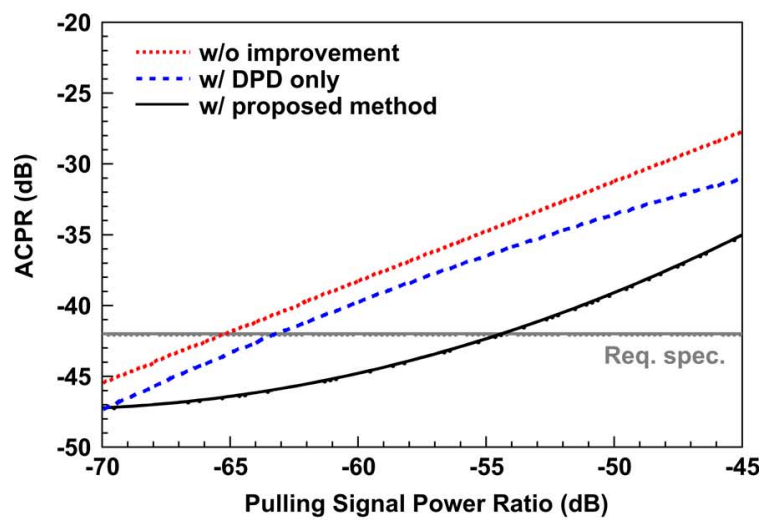

(b)

Fig. 6. Experimental results for the experimental DCT when delivering a 1.98-GHz cdma2000 1x QPSK-modulated signal with a channel bandwidth of $1.25 \mathrm{MHz}$ and an OBO of $6 \mathrm{~dB}$. (a) EVM. (b) ACPR.

eration of adding the second-point VCO modulation frequency, the LO output frequency can be expressed as

$$
\begin{aligned}
\omega_{\mathrm{LO}}(t) \approx & \omega_{c}+K_{v} V_{t}(t)+K_{\mathrm{mod}} V_{\mathrm{mod}}(t)-\omega_{\mathrm{LR}}(t) \alpha(t) \\
= & \omega_{c}+K_{v} V_{t}(t)+\left(K_{\mathrm{mod}} G_{\text {mixer }}-\frac{\omega_{c}}{2 Q E_{\mathrm{osc}}}\right) \\
& \times E_{\mathrm{inj}}(t)\left(\theta_{i}(E(t))+\phi_{m}(t)\right) \\
& -\omega_{\mathrm{LR}}(t)\left(\Phi\left(t-\tau_{0}\right)-\Phi(t)\right)
\end{aligned}
$$

where $\Phi(t)=\phi_{\mathrm{LO}}(t)+\phi_{m}(t)+\theta_{i}(E(t))$. Assume that the pulling signal injection path delay $\tau_{o}$ is sufficiently small when compared to the system modulation symbol time $\tau_{s}$. This can be regarded as true because $\tau_{o}$ is about $20 \mathrm{~ns}$ for our experimental case, while $\tau_{s}$ is around 800 and $260 \mathrm{~ns}$ for the cdma2000 1x and W-CDMA systems, respectively. Therefore, the last term $\omega_{\mathrm{LR}}(t)\left(\Phi\left(t-\tau_{0}\right)-\Phi(t)\right)$ in (19) can be disregarded. Finally, the concerned LO spectral impurity caused by the LO pulling effect can be eliminated while satisfying the following cancellation condition:

$$
K_{\text {mod }} G_{\text {mixer }}=\frac{\omega_{c}}{2 Q E_{\text {osc }}} .
$$

Secondly, if the open-loop DPD method fails to reduce the PA nonlinear distortion, especially the serious PA's AM-PM distortion due to the thermal noise or memory effect, we recommend

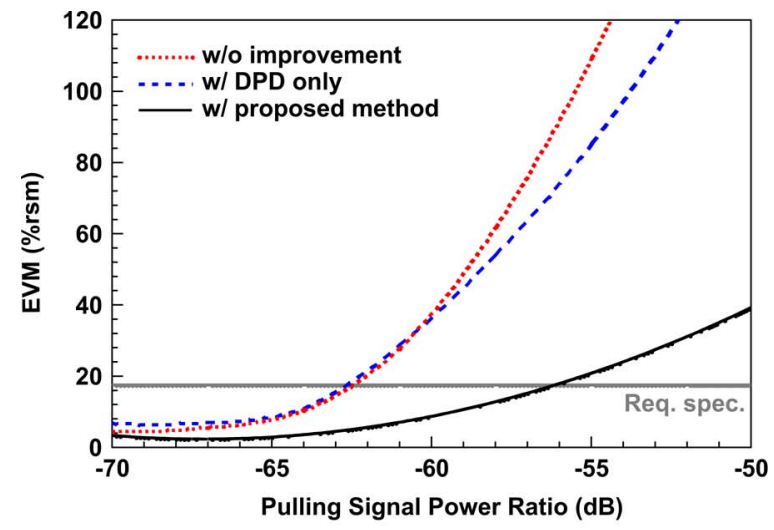

(a)

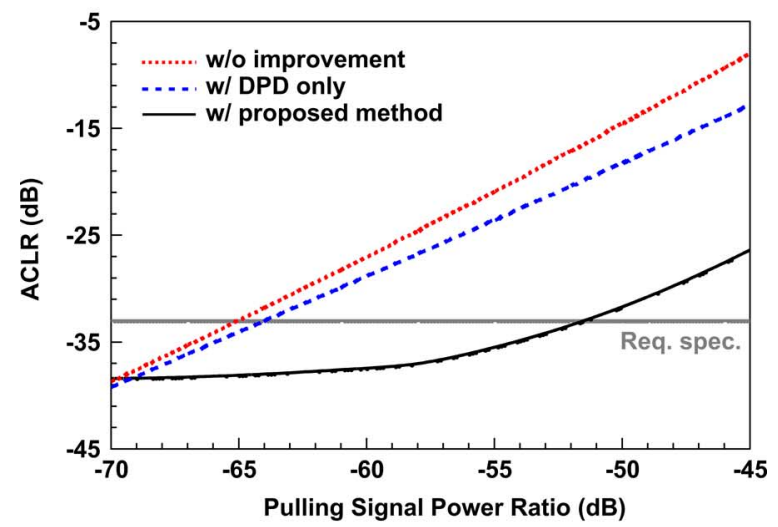

(b)

Fig. 7. Experimental results for the proposed DCT when delivering a 1.98-GHz W-CDMA QPSK-modulated signal with a channel bandwidth of $3.84 \mathrm{MHz}$ and an OBO of $8 \mathrm{~dB}$. (a) EVM. (b) ACLR.

that designers should only preserve the BDP's AM-AM predistortion function, while replacing the original AM-PM compensation mechanism with the proposed second-point VCO modulation approach. Notably, in this suggested approach, the PA's AM-PM distortion function $\theta_{i}(E(t))$ is extracted first from the low-frequency mixing product of the PA's input signal $S_{\mathrm{PA} \_ \text {in }}(t)$ and output signal $S_{\mathrm{TX}}(t)$, as noted in Fig. 4 , then a closed feedback loop is established to mainly compensate for the PA's $\mathrm{AM}-\mathrm{PM}$ distortion.

\section{Inner Self-Injection}

Above analyses also reveal that, if the pulling signal injection path delay $\tau_{o}$ cannot be ignored, the residual spectral impurity term $\omega_{\mathrm{LR}}(t)\left(\Phi\left(t-\tau_{0}\right)-\Phi(t)\right)$ in (19) may significantly degrade the improvement on the transmitted signal quality ultimately. Additionally, some critical PA physical defect issues are too complex to be discussed with the LO pulling effect, which may decrease the improvement of the above cancellation approach. Therefore, an inner self-injection loop is additionally introduced in the proposed DCT to further improve the transmitted signal quality.

Based on the oscillator self-injection-locking concept in [25] and [26], our previous work [11] developed an inner self-injection approach to mitigate the LO pulling effect in a constant envelope modulation system. Figs. 4 and 5 indicate that, in the inner self-injection loop, a part of the pulled LO output signal 


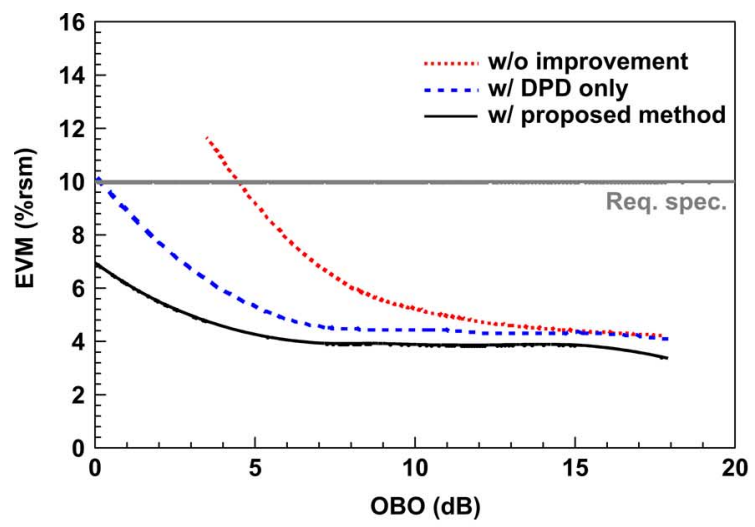

(a)

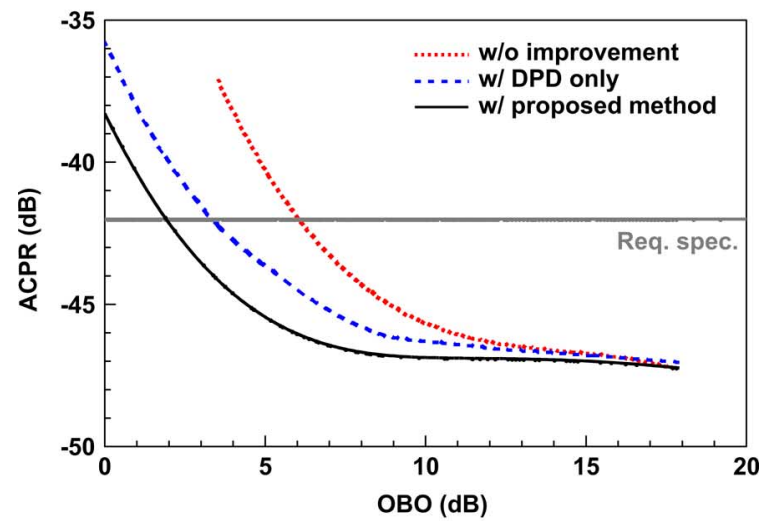

(b)

Fig. 8. Experimental results for the proposed DCT when delivering a 1.98-GHz cdma2000 1x QPSK-modulated signal with a channel bandwidth of $1.25 \mathrm{MHz}$ and a pulling signal power ratio of $-65 \mathrm{~dB}$. (a) EVM. (b) ACPR.

$S_{\mathrm{LO}}(t)$ is fed back and injected into the VCO via a delay line with time delay $\tau_{i}$, a phase shifter with phase shift $\delta$, and a VGA for voltage amplification. When the inner self-injection approach is applied, the concerned LO spectral impurity can be formulated as

$$
\begin{aligned}
\Delta \omega_{\mathrm{LO}}(t)= & \omega_{\mathrm{LO}}(t)-\omega_{c} \\
\approx & K_{v} V_{t}(t)-\omega_{\mathrm{LR}}(t)\left(\Phi\left(t-\tau_{o}\right)-\Phi(t)\right) \\
& -\omega_{\mathrm{LR} i} \sin \left(\alpha_{i}(t)+\delta\right)
\end{aligned}
$$

where $\omega_{\mathrm{LR} i}$ denotes the inner self-injection locking range. $\alpha_{i}(t) \approx \phi_{\mathrm{LO}}\left(t-\tau_{i}\right)-\phi_{\mathrm{LO}}(t)$ also represents the relation between the instantaneous inner self-injection phase and the LO output phase. By assuming that $\tau_{i}$ is sufficiently small to make the approximation $\alpha_{i}(t) \approx-\Delta \omega_{\mathrm{LO}}(t) \tau_{i}$ and $\delta$ is equal to $180^{\circ},(21)$ can be re-approximated as

$$
\Delta \omega_{\mathrm{LO}}(t)=\frac{K_{v} V_{t}(t)-\omega_{\mathrm{LR}}(t)\left(\Phi\left(t-\tau_{0}\right)-\Phi(t)\right)}{1+\omega_{\mathrm{LR} i} \tau_{i}} .
$$

Equation (22) clearly reveals that the LO spectral impurity can be effectively suppressed by increasing the product of the inner self-injection locking range $\omega_{\mathrm{LR} i}$ and path delay $\tau_{i}$.

\section{RESULTS AND DisCUSSION}

This work demonstrates the effectiveness of the proposed improvement approaches by implementing a prototype DCT with

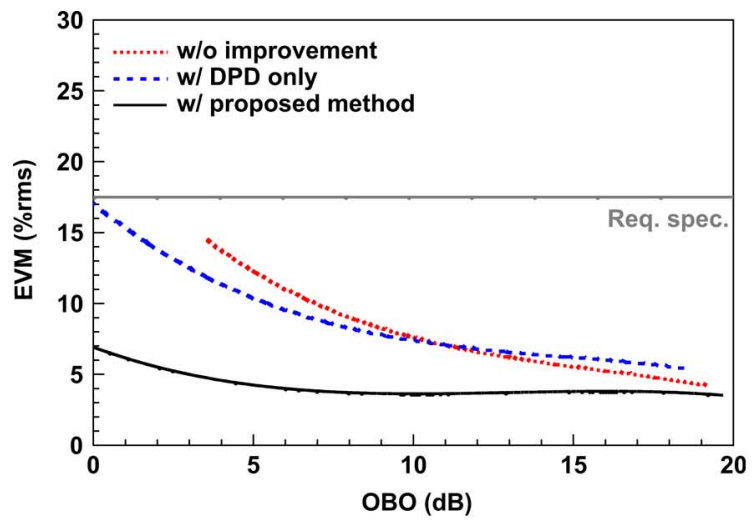

(a)

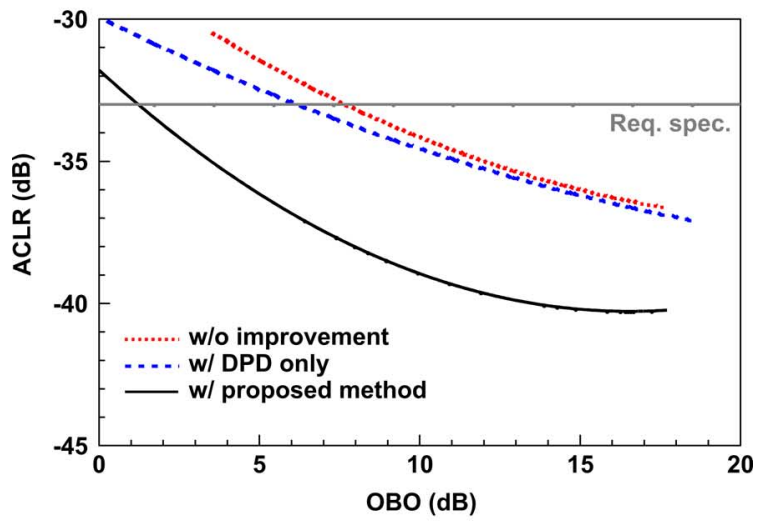

(b)

Fig. 9. Experimental results for the proposed DCT when delivering a 1.98-GHz W-CDMA QPSK-modulated signal with a channel bandwidth of $3.84 \mathrm{MHz}$ and a pulling signal power ratio of $-65 \mathrm{~dB}$. (a) EVM. (b) ACLR.

a system block diagram, as shown in Fig. 4. In the experiments, the $\mathrm{LO}$ is locked at $1.98 \mathrm{GHz}$, delivering an oscillation power of $0 \mathrm{dBm}$. The PA achieves a maximum $\mathrm{CW}$ output power of $29.3 \mathrm{dBm}$. Based on use of the Agilent E4438C VSG applied with Agilent EEsof ADS Ptolemy software, the third-generation $(3 \mathrm{G})$ system modulation signal is generated, including cdma2000 1x QPSK-modulated signals with a PAPR of about $6 \mathrm{~dB}$ [27] and one-frequency-aggregation (1-FA) W-CDMA QPSK-modulated signals with a PAPR of about $8 \mathrm{~dB}$ [28]. Sections IV-A and IV-B validate the improved effectiveness of the transmitted signal quality in terms of EVMs, ACPRs, or adjacent channel leakage ratios (ACLRs), spectral regrowth, and average efficiencies under various injection conditions and modulation systems

\section{A. Ability to Resist PA Distortion and LO Pulling}

Fig. 6(a) and (b) summarizes the experimental results of EVMs and ACPRs, respectively, under various pulling signal power ratios when the DCT delivers a $1.98 \mathrm{GHz}$ cdma2000 1x QPSK-modulated signal with a channel bandwidth of $1.25 \mathrm{MHz}$ and an $\mathrm{OBO}$ of $6 \mathrm{~dB}$. These figures include results achieved by using various improvement approaches, where the dotted lines in red (in online version), broken lines in blue (in online version), and solid lines in black denote the measurement results of without improvements, only the DPD method, and with the proposed approaches, respectively. Additionally, 


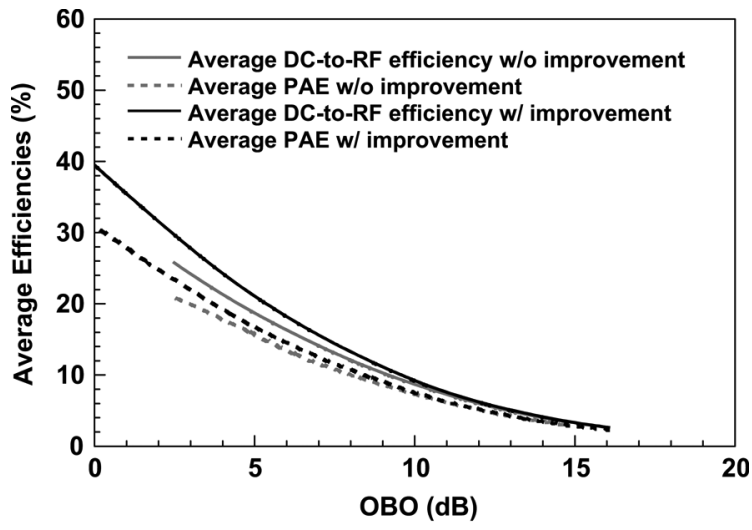

(a)

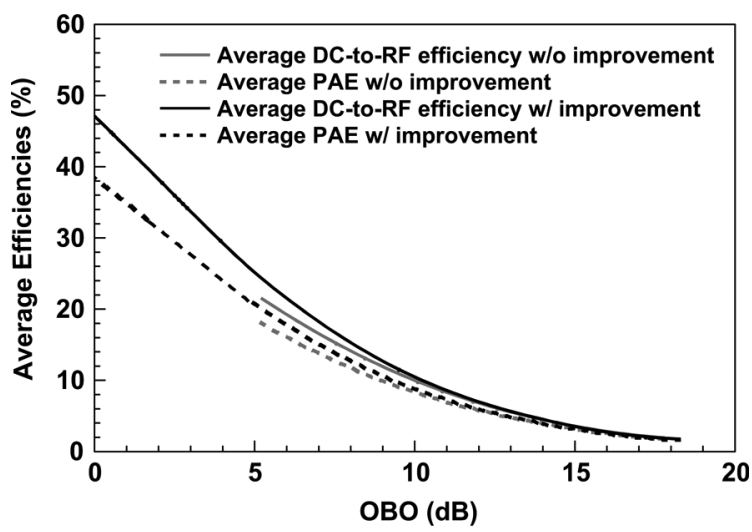

(b)

Fig. 10. Comparison of measured average efficiencies with respect to OBO for the proposed DCTs operating with a pulling signal power ratio of $-65 \mathrm{~dB}$. (a) Delivering a 1.98-GHz cdma2000 1x QPSK-modulated signal with a channel bandwidth of $1.25 \mathrm{MHz}$. (b) Delivering a 1.98-GHz W-CDMA QPSK-modulated signal with a channel bandwidth of $3.84 \mathrm{MHz}$.

the solid lines in gray depict the EVM and ACPR minimum specifications for the applied system. Both EVM and ACPR significantly decrease with an increasing pulling signal power ratio value. Measurement results indicate that the proposed approach improves the EVM by $15.6 \%$ and ACPR by $7.7 \mathrm{~dB}$ when the pulling signal power ratio equals $-55 \mathrm{~dB}$.

Moreover, to emphasize the advantages of wideband and adaptability, Fig. 7(a) and (b) compares the EVMs and ACLRs, respectively, under various pulling signal power ratios when the DCT delivers a 1.98-GHz W-CDMA QPSK-modulated signal with a channel bandwidth of $3.84 \mathrm{MHz}$ and an OBO of $8 \mathrm{~dB}$. According to this figure, a significant improvement on transmitted signal quality is achieved on EVM and ACPR of $85 \%$ and $14.65 \mathrm{~dB}$, respectively, when the pulling signal power ratio equals $-55 \mathrm{~dB}$. According to Figs. 6 and 7, the experimental results verify that the proposed approaches can effectively diminish the combined effects of PA distortion and LO pulling in a time-varying envelope modulation system.

\section{B. Ability to Enhance DCT Linearity and Average Efficiency}

This work also demonstrates the effectiveness of the proposed approaches in enhancing the DCT performance. Figs. 8 and 9 compare the EVMs [see Figs. 8(a) and 9(a)] and ACPRs/

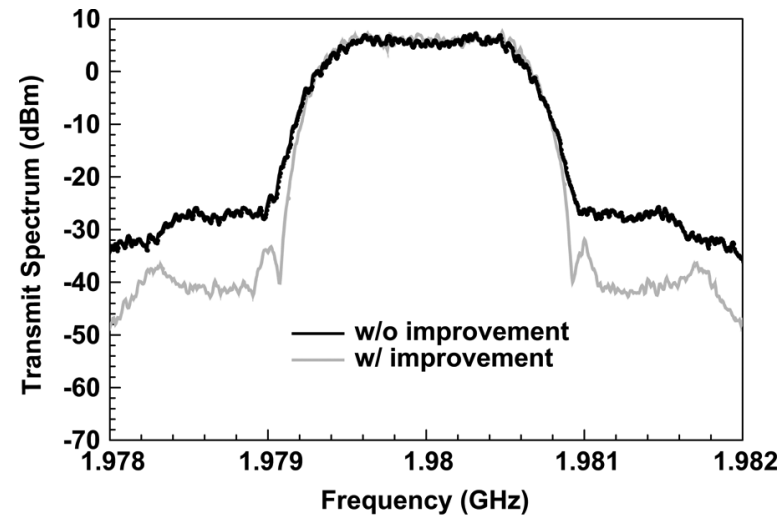

(a)

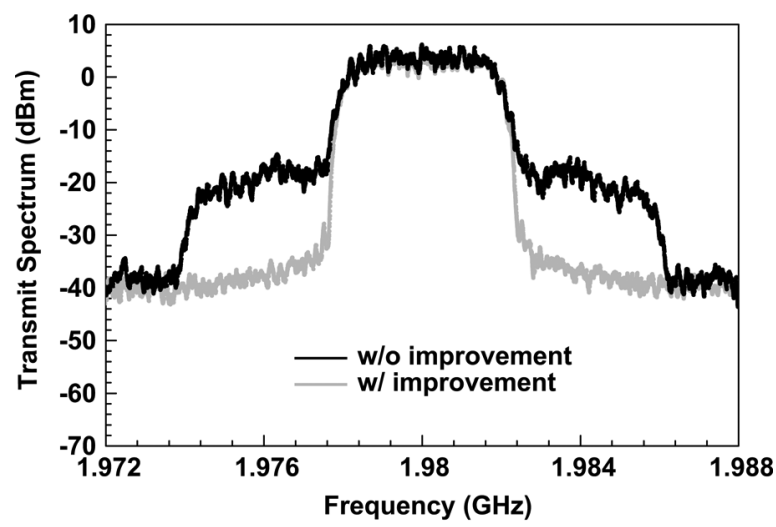

(b)

Fig. 11. Experimental results of transmit spectra for the proposed DCTs operating with an $\mathrm{OBO}$ of $2.5 \mathrm{~dB}$ and a pulling signal power ratio of $-65 \mathrm{~dB}$. (a) $1.98-\mathrm{GHz}$ cdma2000 1x QPSK-modulated signal with a channel bandwidth of 1.25 MHz. (b) 1.98-GHz W-CDMA QPSK-modulated signal with a channel bandwidth of $3.84 \mathrm{MHz}$.

ACLRs [see Figs. 8(b) and 9(b)] under a constant pulling signal power ratio of $-65 \mathrm{~dB}$, but with different OBOs for a DCT that delivers a 1.98-GHz cdma2000 1x QPSK-modulated signal and a W-CDMA QPSK-modulation signal, respectively. In comparison with the conventional DPD methods, the proposed approaches markedly improve in modulation quality. Under the constraint that satisfies the ACPR/ACLR specifications, the proposed approaches can increase the OBO by about 4 and $6.5 \mathrm{~dB}$ for cdma2000 1x system and W-CDMA system, respectively.

Fig. 10 shows the measured average efficiencies with respect to OBO under a constraint that ACPRs/ACLRs must meet the system specifications. In Fig. 10(a), the black lines and gray lines denote the average efficiencies of with and without improvement, respectively, in the cdma2000 1x system. These results indicate that the average DC-to-RF efficiency and average power-added efficiency (PAE) improve significantly, ranging from $25.9 \%$ to $39.5 \%$ and $20.9 \%$ to $30.8 \%$, respectively. Moreover, in Fig. 10(b), a similar improvement on average efficiencies can also be found when the DCT delivers a W-CDMA modulation signal, which ranges from $21.6 \%$ to $47.1 \%$ at average DC-to-RF efficiency and ranges from $17.9 \%$ to $38.2 \%$ at average PAE.

Experimental results show that the proposed approaches have more obvious improvements on the W-CDMA system than on 
the cdma2000 1x system. Above comparisons verify the inference in Section III that the open-loop DPD method fails to accurately compensate for the PA nonlinear distortion due to the lack of adaptive self-correcting mechanism. This failure explains the degraded improvement with an increasing system modulation bandwidth. However, the proposed combined approaches can overcome this limitation.

Finally, Fig. 11(a) and (b), respectively, compares the transmit spectra measurement results for the cdma2000 1x and W-CDMA DCTs when operating at an OBO of $2.5 \mathrm{~dB}$ and a pulling signal power ratio of $-65 \mathrm{~dB}$. In these figures, the solid lines in black and dotted lines in gray denote the DCT output spectrum of without and with improvement, respectively. Above results reveal that the proposed DCT possesses a significant improvement in spectral regrowth, while achieving a high efficiency simultaneously.

In contrast to existing adaptive DPD methods, the combined approach of the open-loop DPD and analog RF feedback technique in this study can reduce DSP computation time and requirements, thus benefiting higher data-rate modulation schemes. However, the RF complexity is undoubtedly increased. Therefore, we recommend that designers use our proposed approach for high data-rate wireless communication systems that suffer from the combined effects of PA distortion and LO pulling.

\section{CONCLUSION}

This paper has presented the combined effects of PA distortion and LO pulling on a DCT in a time-varying envelope modulation system. The proposed theoretical model and analysis approaches can account for and forecast the degraded transmitted signal quality, including the factors such as EVMs, ACPRs, and spectral regrowth. To mitigate the adverse effects on the above factors, an innovative approach, capable of incorporating a baseband DPD method, a second-point VCO modulation approach, and an inner self-injection technique, is developed to improve the deteriorated transmit signal quality. Additionally, the proposed approaches also provide an excellent PA linearization capability and are successfully identified in a case-study application to the cdma2000 1x and W-CDMA systems. Consequently, the proposed DCT is characterized by its superior linearity, high average efficiency, and effective anti-LO pulling properties for application to a high PAPR wireless system. Efforts are underway to extend the proposed approach and related results to more complex modulation systems, such as worldwide interoperability for microwave access (WiMAX) and long term evolution (LTE).

\section{REFERENCES}

[1] B. Razavi, "Challenges in portable RF transceiver design," IEEE Circuits Device Mag., vol. 12, no. 5, pp. 12-25, Sep. 1996.

[2] B. Razavi, "RF transmitter architectures and circuits," in Proc. IEEE Custon Integr. Circuits Conf., Aug. 1999, pp. 197-204.

[3] S. C. Cripps, Advanced Techniques in RF Power Amplifier Design. Norwood, MA: Artech House, 2002.

[4] P. B. Kenington, High-Linearity RF Amplifier Design. Norwood, MA: Artech House, 2000.

[5] S. P. Stapleton, "Digital predistortion of power amplifiers," Agilent Technol. Inc., Santa Clara, CA, Sep. 1, 2005. [Online]. Available: http://www.agilent.com
[6] J. K. Cavers, "Amplifier linearization using a digital predistorter with fast adaptation and low memory requirements," IEEE Trans. Veh. Technol., vol. 39, no. 4, pp. 374-382, Nov. 1990.

[7] L. Sundstrom, M. Haulkner, and M. Johanson, "Quantization analysis and design of a digital predistortion linearizer for RF power amplifier," IEEE Trans. Veh. Technol., vol. 45, no. 4, pp. 707-719, Nov. 1996.

[8] B. Razavi, "A study of injection locking and pulling in oscillators," IEEE J. Solid-State Circuits, vol. 39, no. 9, pp. 1415-1424, Sep. 2004.

[9] C.-J. Li, C.-H. Hsiao, F.-K. Wang, T.-S. Horng, and K.-C. Peng, "A rigorous analysis of a phased-locked oscillator under injection," IEEE Trans. Microw. Theory Tech., vol. 58, no. 5, pp. 1391-1400, May 2010.

[10] C.-H. Hsiao, C.-J. Li, F.-K. Wang, T.-S. Horng, and K.-C. Peng, "Study of direct-conversion transmitter pulling effects in constant envelope modulation systems," in IEEE MTT-S Int. Microw. Symp. Dig., May 2010, pp. 1174-1177.

[11] C.-H. Hsiao, C.-J. Li, F.-K. Wang, T.-S. Horng, and K.-C. Peng, "Analysis and improvement of direct-conversion transmitter pulling effects in constant envelope modulation systems," IEEE Trans. Microw. Theory Tech., vol. 58, no. 12, pp. 4137-4146, Dec. 2010.

[12] I. Bashir, R.-B. Staszewski, O. Eliezer, B. Banerjee, and P.-T. Balsara, "A novel approach for mitigation of RF oscillator pulling in a polar transmitter," IEEE J. Solid-State Circuits, vol. 46, no. 2, pp. 403-415, Feb. 2011

[13] C.-H. Hsiao, C.-T. Chen, T.-S. Horng, and K.-C. Peng, "Direct-conversion transmitter with resistance to local oscillator pulling in nonconstant envelope modulation systems," in IEEE MTT-S Int. Microw. Symp. Dig., May 2011, pp. 1174-1177.

[14] S. Mendel, C. Vogel, and N. Da Dalt, "A phase-domain all-digital phaselocked loop architecture without reference clock retiming," IEEE Trans. Circuits Syst. II, Exp. Briefs, vol. 56, no. 11, pp. 860-864, Nov. 2009.

[15] J. Dominguez, A. Suarez, and S. Sancho, "Semi-analytical formulation for the analysis and reduction of injection-pulling in front-end oscillators," in IEEE MTT-S Int. Microw. Symp. Dig., Jun. 2009, pp. $1589-1592$

[16] C.-T. Chen, C.-J. Li, T.-S. Horng, J.-K. Jau, and J.-Y. Li, "Design and linearization of class-E power amplifier for non-constant envelope modulation," IEEE Trans. Microw. Theory Tech., vol. 57, no. 4, pp 957-964, Apr. 2009.

[17] R. Adler, "A study of locking phenomena in oscillators," Proc. IRE, vol. 34, no. 6, pp. 351-357, Jun. 1946.

[18] S. P. Stapleton, G. S. Kandola, and J. K. Cavers, "Simulation and analysis of an adaptive predistorter utilizing a complex spectral convolution," IEEE Trans. Veh. Technol., vol. 41, no. 4, pp. 387-394, Nov. 1992.

[19] M. Faulkner and M. Johansson, "Adaptive linearization using predistortion-Experimental results," IEEE Trans. Veh. Technol., vol. 43, no. 2, pp. 323-332, May 1994.

[20] J. Kim, C. Park, J. Moon, and B. Kim, "Analysis of adaptive digital feedback linearization techniques," IEEE Trans. Circuits Syst. I, Reg. Papers, vol. 57, no. 2, pp. 345-354, Feb. 2010.

[21] S. Chung, J.-W. Holloway, and J.-L. Dawson, "Open-loop digital predistortion using Cartesian feedback for adaptive RF power amplifier linearization," in IEEE MTT-S Int. Microw. Symp. Dig., Jun. 2007, pp. $1449-1452$.

[22] K. J. Muhonen, M. Kavehrad, and R. Krishnamoorthy, "Look-up table techniques for adaptive digital predistortion: a development and comparison," IEEE Trans. Veh. Technol., vol. 49, no. 5, pp. 1995-2002, Sep. 2000

[23] C. H. Lin et al., "Dynamically optimum lookup-table spacing for power amplifier predistortion linearization," IEEE Trans. Microw. Theory Tech., vol. 54, no. 5, pp. 2118-2127, May 2006.

[24] H. Ku and J.-S. Kenney, "Behavioral modeling of nonlinear RF power amplifiers considering memory effects," IEEE Trans. Microw. Theory Tech., vol. 51, no. 12, pp. 2495-2504, Dec. 2003.

[25] H.-C. Chang, "Stability analysis of self-injection-locked oscillators," IEEE Trans. Microw. Theory Tech., vol. 51, no. 9, pp. 1989-1993, Sep. 2003.

[26] A. Suarez and F. Ramirez, "Analysis of stabilization circuits for phase-noise reduction in microwave oscillators," IEEE Trans. Microw. Theory Tech., vol. 53, no. 9, pp. 2743-2751, Sep. 2005.

[27] B. Yuen, "Crest factor invariant RF power detector," Texas Instrum. Incorporated, Dallas, TX, Appl. Note 1434, 2006.

[28] J. Moon, J. Son, J. Kim, I. Kim, S. Jee, Y. Y. Woo, and B. Kim, "Doherty amplifier with envelope tracking for high efficiency," in IEEE MTT-S Int. Microw. Symp. Dig., May 2010, pp. 1086-1089. 


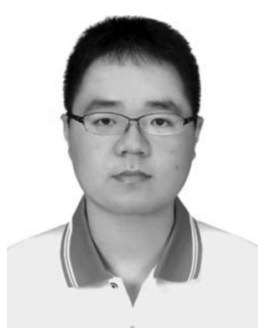

Chieh-Hsun Hsiao (S'10) was born June 5, 1984, in Kaohsiung, Taiwan. He received the B.S.E.E. and M.S.E.E. degrees from the National Sun Yat-Sen University, Kaohsiung, Taiwan, in 2006 and 2008, respectively, and is currently working toward the $\mathrm{Ph} . \mathrm{D}$. degree in electrical engineering at National Sun Yat-Sen University. His doctoral research concerns phase- and injection-locked oscillators.

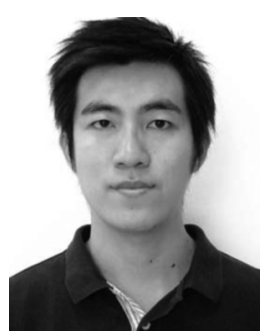

Chi-Tsan Chen (S'07) was born in Taichung, Taiwan, in 1982. He received the B.S.E.E. and M.S.E.E degrees from National Sun Yat-Sen University, Kaohsiung, Taiwan, in 2005 and 2007, respectively, and is currently working toward the $\mathrm{Ph} . \mathrm{D}$. degree in electrical engineering at National Sun Yat-Sen University.

His current research interests include RF PAs, highly efficient and linear transmitter design, and low-power transceivers.

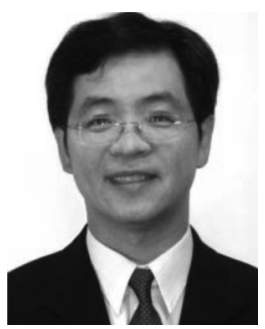

Tzyy-Sheng Horng (S'88-M'92-SM'05) was born in Taichung, Taiwan, on December 7, 1963. He received the B.S.E.E. degree from National Taiwan University, Taipei, Taiwan, in 1985, and the M.S.E.E. and Ph.D. degrees from the University of California at Los Angeles (UCLA), in 1990 and 1992, respectively.

Since August 1992, he has been with the Department of Electrical Engineering, National Sun Yat-Sen University, Kaohsiung, Taiwan, where he was the Director of the Telecommunication Research and De- velopment Center (2003-2008) and Director of the Institute of Communications Engineering (2004-2007), and where he is currently a Professor and the Advanced Semiconductor Engineering Inc. (ASE Inc.) Chair Professor. He has authored or coauthored over 100 technical publications published in IEEE journals and conferences proceedings. He holds over ten patents. His research interests include RF and microwave ICs and components, RF signal integrity for wireless system-in-package, digitally assisted RF technologies, and green radios for cognitive sensors and Doppler radars.

Dr. Horng has served on several Technical Program Committees of international conferences including the International Association of Science and Technology for Development (IASTED) International Conference on Wireless and Optical Communications, the IEEE Region 10 International Technical Conference, the IEEE International Workshop on Electrical Design of Advanced Packaging and Systems, the Asia-Pacific Microwave Conference, the IEEE Radio and Wireless Symposium, and the Electronic Components and Technology Conference. He was the recipient of the 1996 Young Scientist Award presented by the International Union of Radio Science, the 1998 Industry-Education Cooperation Award presented by the Ministry of Education, Taiwan, and the 2010 Distinguished Electrical Engineer Award presented by the Chinese Institute of Electrical Engineering, Taiwan.

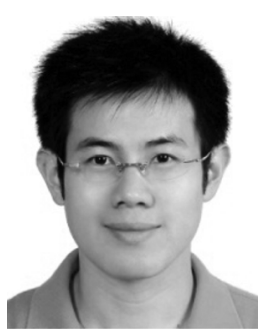

Kang-Chun Peng (S'00-M'05) was born February 18, 1976, in Taipei, Taiwan. He received the B.S.E.E., M.S.E.E., and Ph.D. degrees from National Sun Yat-Sen University, Kaohsiung, Taiwan, in 1998,2000 , and 2005, respectively.

$\mathrm{He}$ is currently an Assistant Professor with the Department of Computer and Communication Engineering, National Kaohsiung First University of Science and Technology, Kaohsiung, Taiwan. His current research interests are in the area of delta-sigma modulation techniques, low-noise PLLs, low-power VCOs, and modulated frequency synthesizers. 